\title{
Toroidal Circular Dichroism
}

\author{
T. A. Raybould, ${ }^{1, *}$ V. A. Fedotov, ${ }^{1}$ N. Papasimakis,${ }^{1}$ I. Kuprov ${ }^{2}$ \\ I. J. Youngs, ${ }^{3}$ W. T. Chen, ${ }^{4}{ }^{\dagger}$ D. P. Tsai, ${ }^{4,5}$ and N. I. Zheludev ${ }^{1,6}$ \\ ${ }^{1}$ Optoelectronics Research Centre, University of Southampton, SO17 1BJ, United Kingdom \\ ${ }^{2}$ School of Chemistry, University of Southampton, SO17 1BJ, United Kingdom \\ ${ }^{3}$ DSTL, Salisbury, United Kingdom \\ ${ }^{4}$ Department of Physics, National Taiwan University, Taipei, 1061\%, Taiwan \\ ${ }^{5}$ Research Center for Applied Sciences, Academia Sinica, Taipei 115, Taiwan \\ ${ }^{6}$ Centre for Disruptive Photonic Technologies, TPI, Nanyang Technological University, Singapore
}

(Dated: May 31, 2016)

We demonstrate that the induced toroidal dipole, represented by currents flowing on the surface of a torus, makes a distinct and indispensable contribution to circular dichroism. We show that toroidal circular dichroism supplements the well-known mechanism involving electric dipole and magnetic dipole transitions. We illustrate this with rigorous analysis of the experimentally measured, polarization-sensitive transmission spectra of an artificial metamaterial, constructed from elements of toroidal symmetry. We argue that toroidal circular dichroism shall be found in large biomolecules with elements of toroidal symmetry and should be taken into account in the interpretation of circular dichroism spectra of organics.

Chirality, i.e. the property by which a pattern cannot be superimposed with its mirror image [1], is a basic characteristic of structured matter at all scales, from the cosmological to the molecular. It enters fundamental questions, such as the homochirality observed in life [2] and the parity violation within the Standard Model of physics [3, 4]. An ubiquitous manifestation of chirality is optical activity, an optical effect characterized by the rotation of the polarization plane of linearly polarized light propagating through a chiral medium, and preferential transmission of circularly polarized light of a particular handedness (circular dichroism) if the medium is dissipative. Observed more than 150 years ago by Louis Pasteur on solutions of tartaric acid [5], optical activity occupies a central position in the diagnostic methodology of many scientific disciplines [6], where it provides means of obtaining information about the microscopic structure and electromagnetic excitations of a medium from its far-field scattering properties. An examination of optical activity is invaluable, for example, in the biosciences where it enables deduction of the conformation of proteins in a dynamic fashion [7], or in the study of magnetic phenomena in strongly correlated electronic systems [8].

Optical activity is typically described within the dipole approximation of the dynamic multipole expansion [6], where the interaction of an incident wave with induced, collinear electric and magnetic dipoles affects the polarization state of the wave upon transmission. Certain combinations of electric dipole and other higher-order multipoles (most notably electrical quadrupole) can also contribute to optical activity [9, 10], although such contributions are usually considered negligible, especially in isotropic media $[11,12]$. It has been suggested [13] that optical activity could arise even in the absence of an electric dipolar response due to the excitation of toroidal multipoles - an elusive part of the dynamic multipole response [14-16].

The toroidal dipole is a localized electromagnetic excitation, distinct from the electric and magnetic dipoles. While the electric dipole can be understood as a pair of opposite charges and the magnetic dipole as a current loop, the toroidal dipole corresponds to currents flowing on the meridians of a torus. Toroidal dipoles provide physically significant contributions to the basic characteristics of matter including absorption and dispersion. Recent experimental observations of resonant toroidal dipole excitations in metamaterials and the discovery of anapoles, non-radiating charge-current configurations involving toroidal dipoles has attracted considerable attention to the subject (see [17] and references therein).

In this paper we analyse the role of toroidal excitations in the polarization-sensitive response of chiral media. Utilising a structurally-chiral metamaterial, we enhance in a resonant fashion the microscopic toroidal and electric quadrupole responses to circularly polarized radiation and show that this previously unexplored combination of dynamically-induced multipole scattering leads to a new mechanism of strong circular dichroism. Our results challenge the common interpretation of circular dichroism based on the electric dipole-magnetic dipole approximation and enhance its capabilities as a diagnostic tool.

The metamaterial used for this study has been derived from the structure previously shown to support a strong toroidal dipolar response under linearly polarized excitation [18]. Its metamolecule consists of acluster of four rectangular metallic wire loops embedded in a dielectric slab [Fig. 1(a)]. The loops are arranged about the axis of the metamolecule ( $y$-axis) at separations of $90^{\circ}$ to give a fourfold symmetric structure, where opposite facing loops are separated by a distance $r$. The symmetry is then broken by introducing identical splits to the loops, located 

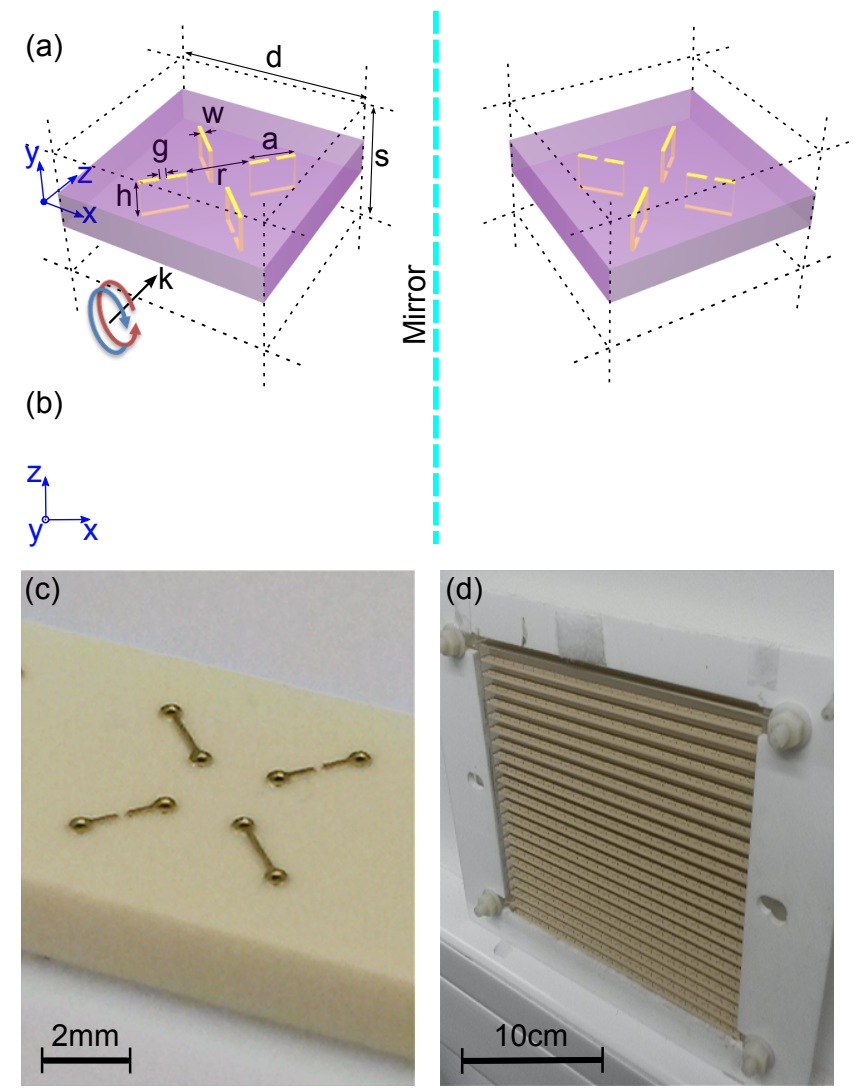

FIG. 1: (a) A schematic of the toroidal metamolecule and its orientation with respect to the metamaterial crystal lattice (indicated by dashed lines). The dimensions are $d=8 \mathrm{~mm}$, $s=7.5 \mathrm{~mm}, h=1.5 \mathrm{~mm}, r=2.44 \mathrm{~mm}, g=w=0.15 \mathrm{~mm}$ and $a=1.8 \mathrm{~mm}$. RCP (red) and LCP (blue) light propagates along the $z$-axis. The individual metamolecules are achiral, as indicated by the mirror image. (b) The metamaterial slab is formed by translating the unit cell along the $x$ and $y$ axes, which imposes structural chirality on the sample. The mirror image shows the enantiomeric form of the metamaterial crystal. (c) Close-up photograph of the fabricated toroidal metamolecule. (d) Metamaterial sample after assembly.

on either the top or the bottom sides of the slab and alternating between adjacent loops. The metamaterial is formed by translating the metamolecule along the $x$ and $y$ axes, resulting in a one-metamolecule thick metamaterial slab with a rectangular unit cell, as shown in Fig. 1(a). Although the individual metamolecules are achiral, the resulting metamaterial crystal is chiral due to the regular arrangement of its metamolecules (structural chirality [19]) and is available in two enantiomeric forms interconnected by a mirror reflection [Fig. 1(b)].

The metamaterial sample was fabricated by etching a $35 \mu \mathrm{m}$ thick copper foil on both sides of a low-loss dielectric PCB laminate Rogers TMM $^{\circledR} 3$ using highresolution photolithography [Fig. 1(c)]. The top and bottom patterns were electrically connected through narrow electroplated holes. All copper tracks were coated

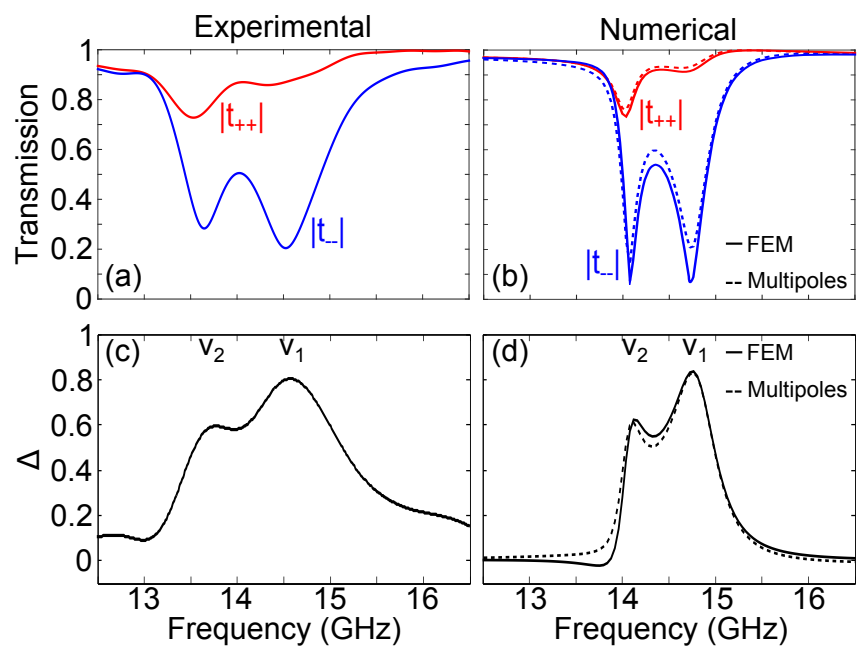

FIG. 2: Measured (left) and calculated (right) circular transmission response of the toroidal metamaterial. (a-b) show the amplitudes of the circularly polarized transmission $t_{++}$and $t_{--}$, while panels (c-d) show circular dichroism $\Delta$. Results calculated using two different methods - finite-element simulation and multipole decomposition of currents are shown by solid and dashed curves, respectively. Pink and navy bands mark the resonances of corresponding conventional $\left(\nu_{1}\right)$ and toroidal $\left(\nu_{2}\right)$ circular dichroism, respectively.

with a $2 \mu \mathrm{m}$ thick layer of gold to prevent oxidization and staining of copper, as well as to reduce Ohmic losses. The assembled metamaterial sample consisted of $24 \times 24$ metamolecules. Its transmission response was experimentally characterized at normal incidence in a microwave anechoic chamber at frequencies in the range 12.5-16.5 $\mathrm{GHz}$ using broadband linearly polarized horn antennas equiped with collimating lenses and a vector network analyzer. Our measurements yielded a complex transmission matrix $t_{i j}(i, j=x, y)$, which fully describes the metamaterial transmission in a linear polarization basis. Using established relations [20], this is converted into the $t_{\alpha \beta}(\alpha, \beta=+,-)$ in a circularly polarized basis, where the + and - denote right $(\mathrm{RCP})$ and left (LCP) circular polarizations respectively.

As indicated by Fig. 2(a), the diagonal elements of the resulting matrix, $t_{++}$and $t_{--}$, are non-identical, indicating the presence of circular dichroism in the transmission response of the metamaterial. The difference between the transmission intensities $\Delta=\left|t_{++}\right|^{2}-\left|t_{--}\right|^{2}$ is a measure of the metamaterial's circular dichroism and it exhibits two resonant bands located at $\nu_{1}=14.6$ and $\nu_{2}=13.6$ $\mathrm{GHz}$, where the dichroism reaches $\sim 0.6$ and $\sim 0.8$, respectively [Fig. 2(b)].

In order to determine the origin of the circular dichroism at these two resonances, we conduct a full-wave simulation of the metamaterial response using a 3D finite element solver (COMSOL 3.5a). The metamaterial array was modelled by placing the toroidal metamolecule in a 

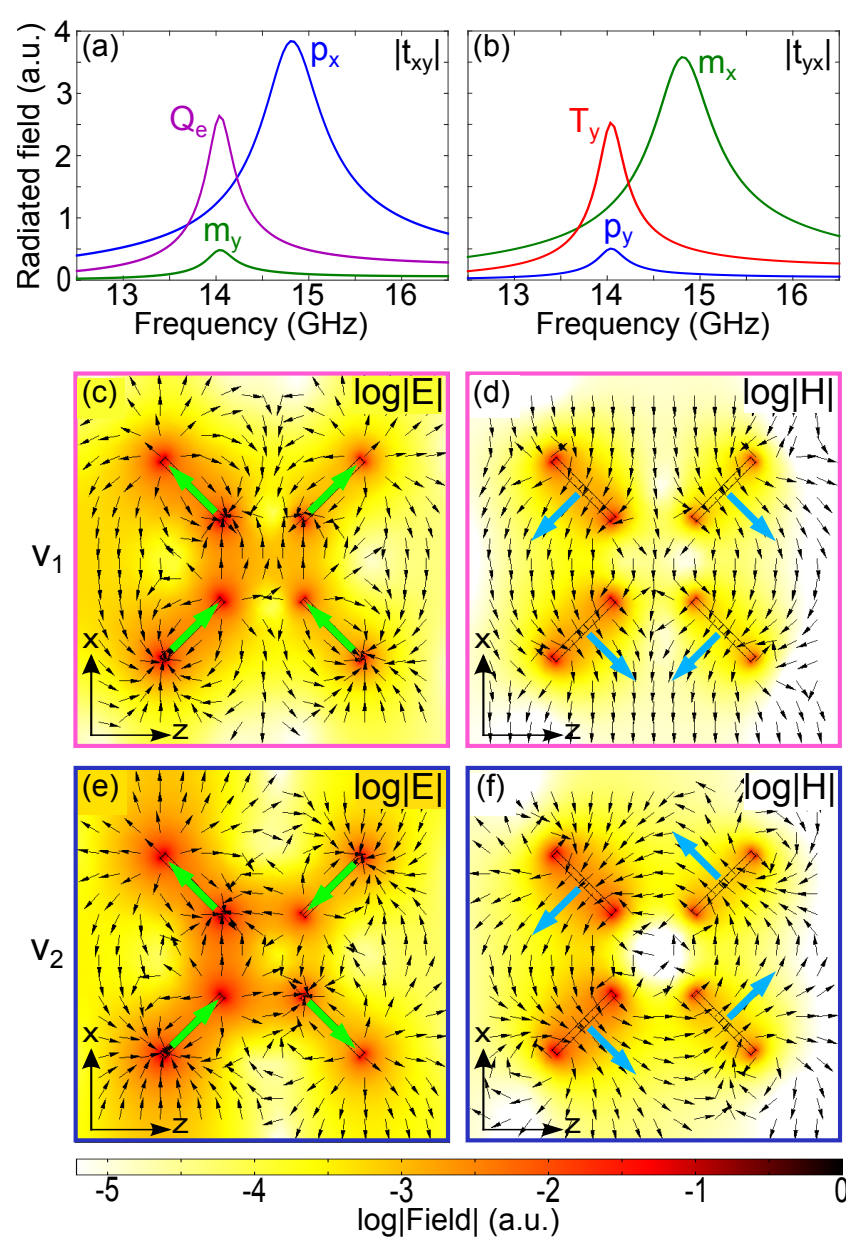

FIG. 3: Amplitudes of the fields radiated by the four most dominant multipole components (electric dipole $\mathbf{p}$, magnetic dipole $\mathbf{m}$, toroidal dipole $\mathbf{T}$ and electric quadrupole $\mathbf{Q}_{\mathbf{e}}$ ) that contribute to polarization conversion response of the metamaterial - (a) $t_{x y}$, and (b) $t_{y x}$. (c-f) give the field distributions $(\log |E|$ and $\log |H|)$ around a metamolecule when excited by LCP light at $\nu_{1}(\mathrm{c}-\mathrm{d})$ and $\nu_{2}$ (e-f). The black arrows give the corresponding $E$ and $H$ field direction. The green (blue) arrows show the orientation of the induced electric (magnetic) dipoles for the individual rings.

rectangular unit cell with periodic boundary conditions imposed along $x$ and $y$ axes [Fig. 1(a)]. The wires of the metallic loops were assumed to be infinitely thin strips of perfect electric conductors. Permittivity of the dielectric slab was set to $\varepsilon=3.45-i 0.007$, which corresponds to Rogers TMM ${ }^{\circledR} 3$ dielectric laminate used in the fabrication of the metamaterial sample [21]. The transmission coefficients of circularly polarized light extracted from this simulation agree well with those observed experimentally [Fig. 2], with the slight discrepancy arising due to fabrication tolerances and uncertainty in the dielectric constant of the PCB laminate.

The microscopic origin of the effect was analysed in terms of multipolar charge-current excitations supported by the metamolecules (analogous to multipolar transitions in molecules). The multipole moments were computed based on spatial distributions of the induced conduction and displacement current densities extracted from the finite element simulation, as per the method described in ref. [18]. The full expressions for the multipole moments can be found in ref. [14]. To simplify the analysis the expansion was truncated at the octopole order for electric and magnetic multipoles, and at the quadrupole order for toroidal multipoles. Following the recently proposed methodology [22], we can directly link the multipolar excitations of individual metamolecules to the far-field response of the entire metamaterial array, and reconstruct the linear and circularly polarized transmission matrices, $t_{i j}$ and $t_{\alpha \beta}$ respectively, from the multipole scattering contributions. The accuracy of this approach is demonstrated by Figs. 2(c-d), where we compare the metamaterial transmission calculated through the multipole decomposition (dashed curves) with that obtained directly from the simulations (solid curves).

For the purpose of the analysis it is sufficient to perform the multipole decomposition of the metamaterial's polarization conversion response, $t_{x y}$ and $t_{y x}$, since these can be regarded as a manifestation of the observed circular dichroism in the linear basis. Indeed, by comparing the linear and circularly polarized transmission matrices [20], it can be shown that circular dichroism $\Delta \propto \Im\left(t_{x y}-t_{y x}\right)$, and so exists only for non-zero and non-identical linear polarization conversion terms. We note that $\left|t_{x y}\right|=\left|t_{y x}\right|$ as dictated by the symmetry of the metamaterial with respect to the propagation direction (z-axis). Figs. 3(a-b) show the results of the multipole decomposition where we retained only six multipole components for clarity, namely $x$-components of electric $\mathbf{p}$ and magnetic $\mathbf{m}$ dipoles at $\nu_{1}$, and electric quadrupole $\mathbf{Q}_{\mathbf{e}}$ and $y$-components of electric, magnetic and toroidal $\mathbf{T}$ dipoles at $\nu_{2}$ (other multipole components are at least one order of magnitude smaller than the dominant ones).

In agreement with the modelled circular dichroism data [Fig. 2(d)], the resonances in the multipole scattering occur at frequencies $\nu_{1}$ and $\nu_{2}$. Figs. 3(a-b) indicate a symmetry to the linear conversion response, as expected from the aforementioned $\left|t_{x y}\right|=\left|t_{y x}\right|$ condition. However, the multipoles contributing to the same resonant features in $t_{x y}$ and $t_{y x}$ are different. This indicates that the observed circular dichroism can be attributed to the simultaneous excitation of pairs of different multipoles. In particular, at $\nu_{1}$ the dominant pair comprises electric and magnetic dipoles with collinear moments $-p_{x}$ and $m_{x}$. Such a combination corresponds to the conventional mechanism of optical activity and hence is considered as a reference case in our further analysis below. At $\nu_{2}$, however, the contributions of $p_{x}$ and $m_{x}$ become only secondary, while the circular dichroism appears to be underpinned by the previously unexplored combination of the toroidal dipole $T_{y}$ and electric quadrupole $Q_{e}$. 
The presence of these multipole pairs can be visually detected in the distributions of electric and magnetic fields inside the unit cell arising under circularly polarized excitation [Figs. 3(c-f)]. At $\nu_{1}$ the field lines of both electric and magnetic fields are seen to align parallel to $x$-axis, which indicates the presence of electric and magnetic dipolar excitations in the metamolecule, with the net dipole moments being collinear and oriented along $x$ axis [Figs. 3(c-d)]. At $\nu_{2}$ magnetic field is confined within a well-defined ring-like area where the field lines form a closed-loop, threading through all individual resonators of the metamolecule [Fig. 3(e)]. Such a magnetic field configuration is unique to the toroidal dipolar excitation with a net dipole moment aligned parallel to the axis of the metamolecule ( $y$-axis), and is formed by poloidal currents flowing in the wire loops of the metamolecules. The distribution of electric field features a pattern similar to that in Fig. 3(c), but with the field lines on the opposite sides of the metamolecule aligned anti-parallel to each other [Fig. 3(f)], which is indicative of an electric quadrupole excitation.

The analysis of the nature of the observed circular dichroism can be further reinforced by examining the role of the identified multipole pairs in shaping the polarization eigenstates of the metamaterial structure. The eigenstates were computed by solving the characteristic equation of the circularly polarized transmission matrix $t_{\alpha \beta}$. Here, $t_{\alpha \beta}$ is again reconstructed from the microscopic multipole response of the system [22]. The polarization eigenstates were characterised in terms of their ellipticity angle $\epsilon$, where $|\epsilon|$ takes values between $\pi / 4$ (circular polarization) and 0 (linear polarization). A positive ellipticity angle defines right handed rotation, while negative - left handed rotation. The two limiting cases, $\epsilon= \pm \pi / 4$ and $\epsilon=0$, represent polarization eigenstates of optically active (chiral) isotropic media and linearly birefringent (achiral) anisotropic media, respectively. The calculated eigenstates of the metamaterial correspond to the intermediate case of counter rotating elliptical polarizations with $\epsilon \approx \pm \pi / 8$ near the resonances, where the ellipticity arises due to anisotropy in the metamaterial structure [Fig. 4(a)].

The roles of the multipoles are assessed by removing in pairs their contributions from the linear conversion response, preserving the symmetry of the conversion and then recalculating the ellipticity angles of the resulting eigenstates. The application of this approach is illustrated in Fig. 4(b) for the reference case. One can see that at $\nu_{1}$ the removal of just $p_{x}$ and $m_{x}$ leads to a decrease of $|\epsilon|$ to approximately $\pi / 60$ for both eigenstates, with their elliptical polarizations collapsing to almost linear states. This significant transition towards a purely anisotropic response confirms that on the microscopic level the observed circular dichroism at $\nu_{1}$ results from electric and magnetic dipole scattering induced in the metamolecules. Using the same approach for the com-
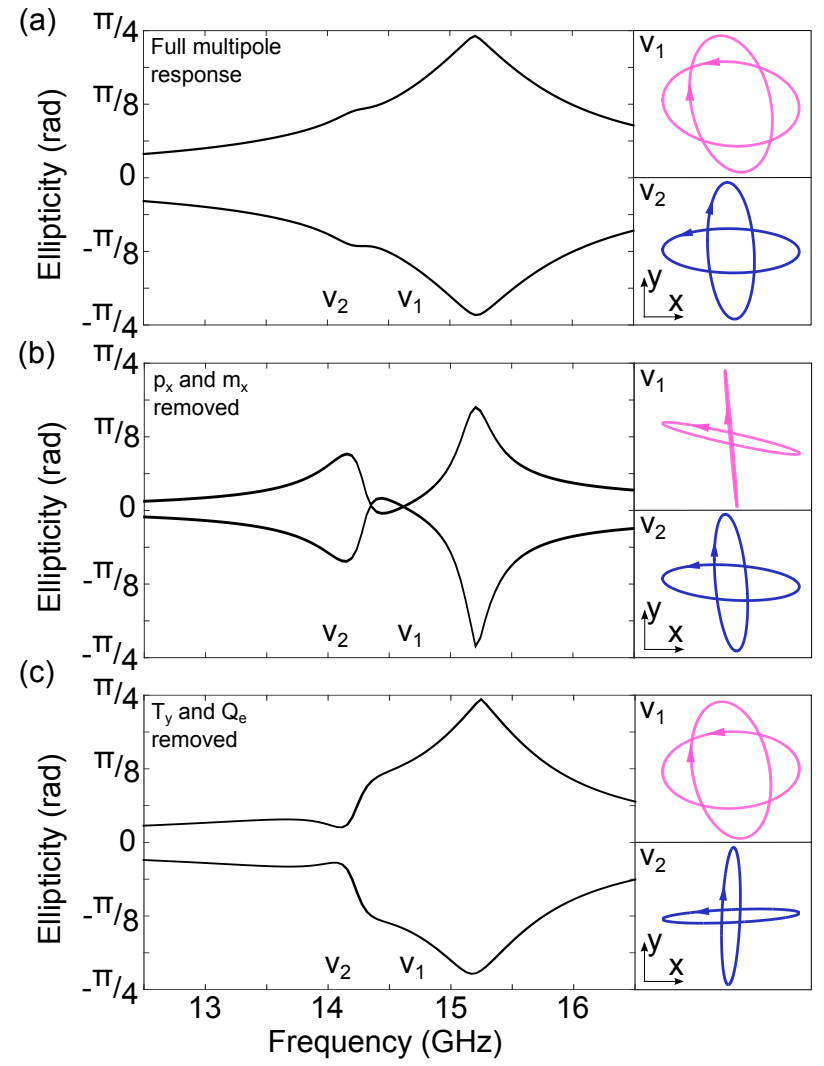

FIG. 4: Ellipticity angles of the metamaterial eigenstates (left) and associated polarization ellipses at $\nu_{1}$ and $\nu_{2}$ (right), calculated based on the multipole expansion of microscopic charge-current excitations supported by toroidal metamolecules. (a) shows the results obtained using the complete multipole set. (b-c) show the results corresponding to pairs $p_{x}-m_{x}$ and $T_{y}-Q_{e}$ being removed from the microscopic response of the metamaterial (solid curves) and compared to the complete multipole response from the upper panel (dashed curves).

bination of $T_{y}$ and $Q_{e}$ we also observed a clear change in the polarization eigenstates but at $\nu_{2}$, where $|\epsilon|$ decreases to approximately $\pi / 30$ [Fig. 4(c)]. These nonvanishing values of $|\epsilon|$ are attributed to the contribution of $p_{x}$ and $m_{x}$ that also impact the circular dichroism at $\nu_{2}$, as indicated by Fig. 4(b). Nevertheless, the resulting modification of the eigenstates at $\nu_{2}$ supports our conclusion that the resonant scattering of the toroidal dipole and electric quadrupole is the dominant mechanism underpinning the circular dichroism at this frequency. The presence of strong toroidal and quadrupole contributions to the circular dichroism of the metamaterial represents a new kind of enantiomeric phenomenon nothing of the kind has been previously observed, even in chemistry where the circular dichroism is a standard analytical technique that reports, for example, on secondary and tertiary structures of proteins [23].

In summary, we have investigated experimentally and 
numerically a structurally chiral metamaterial that exhibits strong circular dichroism and supports a resonant toroidal dipolar response. Analysis of the fields scattered by the charge-current multipoles induced in the metamolecules and the polarization eigenstates of the system, reveals that the metamaterial exhibits both the conventional mechanism of circular dichroism, by way of electric and magnetic dipole transitions; and a previously unobserved form of circular dichroism, underpinned by the resonant excitation of the toroidal dipole and electric quadrupole. We note that metamaterial structures have been utilised to replicate many phenomena which occur in natural media, with metamolecules being analogous to molecules, and conduction currents being analogous to displacement currents and polarization. Hence, this observed multipole combination is anticipated to be the cause of (or at least strongly contribute to) circular dichroism in many other material systems with toroidal topology, uch as large molecules commonly encountered in chemistry and biology, where optical activity is directly linked to the topology of the whole molecule [24-28].

The financial support of DSTL, the Engineering and Physical Sciences Research Council UK, the Leverhulme Trust, the MOE Singapore, the Ministry of Science and Technology of Taiwan, and the Academia Sinica of Taiwan is acknowledged. The authors would like to thank Vassili Savinov for numerous fruitful discussions.

The data from this paper can be obtained from the University of Southampton ePrints research repository: http://dx.doi.org/10.5258/SOTON/XXXXXX

* Electronic address: T.A.Raybould@soton.ac.uk

† Present address: School of Engineering and Applied Sciences, Harvard University, Cambridge, MA, 02138, United States of America

[1] W. T. Kelvin, Baltimore lectures on molecular dynamics and the wave theory of light, (C. J. Clay \& Sons, London, 1904).

[2] Y. Saito and H. Hyuga, Rev. Mod. Phys. 85, 603 (2013).

[3] D. Wang et al, Nature 506, 67 (2014).

[4] T. D. Lee and C. N. Yang, Phys. Rev. 104, 254 (1956).

[5] L. Pasteur, Chim. Phys. 24, 442 (1848).
[6] L. D. Barron, Molecular Light Scattering and Optical Activity, (Cambridge University Press, 2004).

[7] L. Whitmore and B. A. Wallace, Biopolymers 89, 392 (2008).

[8] A. Kirillyuk, A. V. Kimel and T. Rasing, Rev. Mod. Phys. 82, 2731 (2010).

[9] J. A. Schellman, Acc. Chem. Res. 1, 144 (1968).

[10] R. E. Raab and O. L. de Lange, Multipole Theory in Electromagnetism, (Oxford University, New York, 2005).

[11] H. Nakano and H. Kimura, J. Phys. Soc. Jpn. 27, 519 (1969).

[12] A. D. Buckingham and M. B. Dunn, J. Chem. Soc. A 38, 1988 (1971).

[13] N. Papasimakis, V. A. Fedotov, K. Marinov and N. I. Zheludev, Phys. Rev. Lett. 103, 093901 (2009).

[14] E. E. Radescu and G. Vaman, Phys. Rev. E. 65, 046609 (2002).

[15] V. M. Dubovik and V. V. Tugushev, Phys. Rep. 187, 145 (1990).

[16] T. A. Gongora and E. Ley-Koo, Rev. Mex. Fis. E 52, 188 (2006).

[17] N. Papasimakis, V. A. Fedotov, V. Savinov, T. A. Raybould and N. I. Zheludev, Nat. Mat. 15, 263 (2016).

[18] T. Kaelberer, V. A. Fedotov, N. Papasimakis, D. P. Tsai and N. I. Zheludev, Science 330, 1510 (2010).

[19] A. Papakostas, A. Potts, D. M. Bagnall, S. L. Prosvirnin, H. J. Coles and N. I. Zheludev, Phys. Rev. Lett. 90, 107404 (2003).

[20] R. Singh, E. Plum, C. Menzel, C. Rockstuhl, A. K. Azad, R. A. Cheville, F. Lederer, W. Zhang and N. I. Zheludev, Phys. Rev. B 80, 153104 (2009).

[21] Data sheet for Rogers Corporation $\mathrm{TMM}(\mathrm{R})$ laminates [Online], www.rogerscorp.com/acs/products/46/TMM3-Laminates.aspx, Accessed on 08-02-2016

[22] V. Savinov, V. A. Fedotov and N. I. Zheludev, Phys. Rev. B 89, 205112 (2014).

[23] N. Berova, K. J. Nakanishi and R. Woody, Circular dichroism: principles and applications, (Wiley, 2000).

[24] S. Lemak et al, J. Am. Chem. Soc. 135, 17476 (2013)

[25] G. Thorner, J. M. Kiat, C. Bogicevic and I. Kornev, Phys. Rev. B 89, 220103 (2014).

[26] S. Suzuki, Y. Segawa, K. Itami and J. Yamaguchi, Nat. Chem. 7, 227 (2015).

[27] M. J. Urban, P. K. Dutta, P. Wang, X. Duan, X. Shen, B. Ding, Y. Ke and N. Liu, J. Am. Chem. Soc. 138, 5495 (2016).

[28] S. M. Kelly, T. J. Jess, and N. C. Price, Biochim. Biophys. Acta. 10, 119 (2005). 